\title{
Gestion de l'espace et maîtrise des risques de pollution. Une méthode de diagnostic environnemental d'exploitations d'élevage
}

\author{
$\mathrm{J}$ Nocquet \\ ISARA, 31 place Bellecour, 69288 Lyon Cedex 02, France
}

Dans le cadre de l'expérimentation Plan de Développement Durable en zone allaitante de Saône et Loire deux enjeux environnementaux ont été identifiés (Butin et al, 1993, ISARA-Ch d'Agr,71, 23) : la gestion de l'espace (qualités du cadre de vie, paysagère) et la maîtrise des risques de pollution diffuse (nitrates et pesticides) et ponctuelle (stockage des déjections animales).

Le thème environnement constitue un volet complémentaire du diagnostic de l'exploitation agricole proposé précédemment (Nocquet et al, 1994, Cahiers Agricultures, 3, 39-50). Cette méthode de diagnostic privilégie une approche par thèmes, correspondant aux qualités globales (Landais et Gilibert, 1991, URSAD Versailles-Dijon-Mirecourt, INRA, 55 p) d'exploitations d'élevage "respectueuses de l'environnement". Deux thèmes ont été formalisés, en réponse aux deux enjeux environnementaux. Sur la base d'une collecte rapide des informations (une demi-journée d'enquête) et au dire des experts, une liste d'indicateurs hiérarchisés, selon les composants du système "exploitation agricole", a été définie. Cette méthode a été testée sur une quinzaine d'élevages bovins allaitants de race charolaise. Les résultats suivants sont limités à quatre d'entre eux.

Globalement, ces exploitations gèrent bien l'espace. Les éleveurs sont très attachés aux valeurs paysagères, l'entretien du parcellaire est excellent (taille annuelle des haies, bonne maîtrise du pâturage). En outre, l'éleveur A vend des reproducteurs et l'exploitant $C$ a un projet de création de camping à la ferme.

Les situations, concernant la maîtrise des risques de pollution, sont plus contrastées. Le diagnostic met en évidence des problèmes de mise aux normes des bâtiments et de fertilisation azotée des prairies comme du maïs pour les quatre élevages. La situation délicate de l'élevage $C$ s'explique par la part du maïs dans l'assolement (15\%) et par l'importance des surfaces à risques $(40 \%$ de pentes et $90 \%$ de sols très sensibles au lessivage). A court terme, le contraste va s'amplifier, en raison de la capacité de financement de la mise aux normes (élevages $B$ et $C$ ) et du raisonnement de la fertilisation et des traitements phytosanitaires (l'éleveur D raisonne la fertilisation en fonction des terrains et des rendements des cultures, l'éleveur A applique des doses standard).

En conclusion, la méthode de diagnostic environnemental proposée permet de détecter rapidement les forces et les faiblesses de ces élevages, vis-à-vis du respect de l'environnement. Selon le contexte, le diagnostic d'autres enjeux environnementaux peut être formalisé.

\begin{tabular}{|c|c|c|c|c|c|c|c|c|}
\hline \multirow{2}{*}{$\begin{array}{l}\text { Thèmes } \\
\text { Elevages }\end{array}$} & \multicolumn{4}{|c|}{ Maîtrise des risques de pollution } & \multicolumn{3}{|c|}{ Gestion de l'espace } & \\
\hline & A & $B$ & $\mathrm{C}$ & $D$ & A & $B$ & $\mathrm{C}$ & D \\
\hline \multicolumn{9}{|l|}{ Diagnostic : } \\
\hline état actuel & $=$ & $=$ & - & $=$ & + & + & + & + \\
\hline à court terme & $=$ & - & - & + & + & + & + & + \\
\hline
\end{tabular}

